\title{
Automated Purification and Quantification of Oligonucleotides
}

BioTechniques 27:810-823 (October 1999)

K.M. Ivanetich, R.C. Reid, R. Ellison, K. Perry, R. Taylor, M. Reschenberg1, A. Mainieri, D. Zhu, J. Argo, D. Cass and C. Strickland

University of California, San Francisco, CA and ${ }^{1}$ Shimadzu Scientific Instruments, Columbia, MD, USA

\begin{abstract}
We have developed automated methods for the trityl-on purification and quantification of synthetic oligonucleotides. Oligonucleotide purification is by solid-phase extraction cartridges using Amberchrom ${ }^{\circledR}$ CG-50 resin on an XYZ-axis robotic system. Quantification is by $O D_{260 n m}$ using an online $U V$-visible spectrophotometer with sipper. The purification of 20 oligonucleotides requires 5 min of user set-up time, plus 20 min per sample of robot time. For a 15-25mer at the $40 \mathrm{nmol}$ scale of synthesis, the method gives a yield of 2.8 ODs from a load of 10.1 OD, i.e., a $28 \%$ average yield. Oligonucleotides purified by this method have proven to be successful for primers for automated DNA sequencing.
\end{abstract}

\section{INTRODUCTION}

We have developed a rapid and reliable method for the purification of synthetic oligonucleotides. This method is suitable for biotechnology core facilities or laboratories with a throughput of up to approximately ten thousand oligonucleotides per year on one robot. This equates to a capacity of 40 oligonucleotides purified per day or 200 oligonucleotides purified per week.

Reported oligonucleotide purification methods include polyacrylamide gel electrophoresis (PAGE) (7), reverse-phase $(2,3,13)$ or ion-exchange $\operatorname{HPLC}(1,11,13)$ and trityl-on cartridge methods $(5,8,12)$. We previously reported an automated protocol for tritylon oligonucleotide purification using Nensorb $^{\text {TM }}$ Resin (NEN Life Science Products, Boston, MA, USA) (4). Following the withdrawal of Nensorb resin from commercial production, we sought to develop a new automated cartridge method for oligonucleotide purification. The reported method uses Amberchrom ${ }^{\circledR}$ CG-50 Resin (TosoHaas, Montgomeryville, PA, USA) in 4-mL disposable extraction cartridges (DECs). The resin is a polydivinyl benzene polymer (particle size 50-100 $\mu \mathrm{m}$; $0.55-0.75 \mathrm{cc} / \mathrm{cc}$ porosity). The method relies upon differential binding of the tritylated (5'-dimethoxytrityl) and nontritylated oligonucleotides to the resin for purification of full-length synthesis products $(6,8,12)$. In a stepwise manner, the protocol involves column conditioning, binding of the tritylated oligonucleotide and non-tritylated failure sequences, removal of non-tritylated failure sequences, detritylation and elution of the purified product, plus appropriate wash steps. The current protocol includes automated oligonucleotide dilution and mixing. The spectrophotometric quantification of the purified and unpurified oligonucleotides have also been automated. The quantification protocols and the wash procedure for packed columns are performed as separate protocols.

\section{MATERIALS AND METHODS}

Empty 4-mL polypropylene columns (Part No. 1213-1014; Bond Elut reservoirs with frits; Varian Instruments, Santa Clarita, CA, USA) were packed manually with $300 \mathrm{mg}$ Amberchrom CG-50 (medium) resin (Part No. 014564; $100 \mathrm{~g}$ purchased dry) as follows: one frit was pushed to the bottom of the column, $300 \mathrm{mg}$ of dry resin were added to the column by a funnel, and the second frit was pressed onto the top of the resin bed with an inverted Pasteur pipet. A plastic cap with center hole (Part No. 2954665; Gilson, Middleton, WI, USA), which ensures a seal with the robotic needle, was then introduced onto the open end of the column. Finally, the outside of the packed column was wiped with ethanol to remove any loose resin.

The oligonucleotide purification and column washing protocols were performed on one of two ASPEC ${ }^{\mathrm{TM}}$ robots, 
each with a Model 401 Dilutor (10-mL vol; all from Gilson). The configuration of reservoirs and racks on the Gilson ASPEC robots is as follows: Position 0 (Dilutor) which has a capacity of 500 $\mathrm{mL}$ to several liters; the following racks are arrayed from left to right and numbered 1-5: (rack 1) the solvent rack (Part No. B54651), which contains four reservoirs of approximately $250-\mathrm{mL}$ capacity, with one reservoir (bottle 3 ) having a lower luer and attachment to a 500 -mL bottle off the robot bed; (rack 2) a customized rack made from a Code 99 polypropylene rack (original Part No. A46980) milled to hold twenty-one $15 \times 45$-mm oligonucleotide collection vials, plus one reservoir of approximately 300-mL capacity (bottle 5); (rack 3) the sample rack No. 21 for sixty $13 \times 100$-mm test tubes (Part No. AQ46861); and (racks 4 and 5) the liquid solid extraction (LSE) DEC (500 $\mathrm{mg}$ ) rack (capacity 20 samples) (Part No. B54652), containing a polypropylene drain and cover (Part Nos. B54653 and B54657), polypropylene mobile rack for 500-mg DECs (Part No. 54658) and an aluminum rack for collection tubes (Part No. B54659). One ASPEC is connected directly to a Model 1601 UV Spectrophotometer (Shimadzu Scientific Instruments, Columbia, MD, USA) with sipper unit by polyether ether ketone (PEEK) tubing ( $35 \mathrm{~cm} \times 0.020$ in i.d.). The ASPEC was programmed by an Art 3000SX 486 computer using 718 Sample Manager Version 1.3 software (Gilson) and Windows ${ }^{\circledR} 3.1$ (Microsoft, Redmond, WA, USA).

Oligonucleotides were synthesized on Model 394 DNA Synthesizers (PE Biosystems, Foster City, CA, USA) using reagents from PE Biosystems, Glen Research (Sterling, VA, USA) or Prime Synthesis (Aston, PA, USA) and the fast dG-dmf cyanoethyl phosphoramidite chemistry. Typically, oligonucleotides for the protocol were synthesized trityl-on. Oligonucleotides were deprotected for $1 \mathrm{~h}$ at $65^{\circ} \mathrm{C}$ in aqueous ammonium hydroxide (30\% ammonia) (Fisher Scientific, Pittsburgh, PA, USA) and cooled to room temperature.

The robot was loaded as follows: as many as 20 uncapped collection vials, each containing at least $1.5 \mathrm{~mL}$ of deprotected tritylated oligonucleotide

Table 1. Oligonucleotide Purification Data from Automated Amberchrom CG-50 Protocol

\begin{tabular}{|c|c|c|c|c|c|c|c|}
\hline $\begin{array}{l}\text { Synthesis } \\
\text { Scale } \\
(\mu \mathrm{mol})\end{array}$ & $\begin{array}{l}\text { Oligo- } \\
\text { nucleotide } \\
\text { Lengtha }\end{array}$ & & Mean & SD & Min & Max & $\mathbf{N}$ \\
\hline \multirow[t]{3}{*}{0.04} & short & OD On & 9.5 & 1.9 & 3.9 & 15.0 & \\
\hline & & $O D_{\text {Off }}$ & 2.7 & 0.9 & 0.8 & 6.2 & \\
\hline & & \% Yield & 28.2 & 8.1 & 6.0 & 54.3 & 134 \\
\hline \multirow[t]{3}{*}{0.04} & medium & OD $_{\text {On }}$ & 12.7 & 3.2 & 3.6 & 21.2 & \\
\hline & & OD ${ }_{\text {Off }}$ & 3.3 & 1.2 & 1.1 & 7.0 & \\
\hline & & \% Yield & 26.0 & 8.8 & 11.1 & 65.4 & 87 \\
\hline \multirow[t]{3}{*}{0.2} & short & OD $_{\text {On }}$ & 26.7 & 7.9 & 17.2 & 50.0 & \\
\hline & & OD $D_{\text {Off }}$ & 11.2 & 2.7 & 6.5 & 18.4 & \\
\hline & & $\%$ Yield & 43.3 & 9.9 & 29.4 & 63.7 & 23 \\
\hline \multirow[t]{3}{*}{0.2} & medium & OD & 36.0 & 12.1 & 15.0 & 50.7 & \\
\hline & & $\mathrm{OD}_{\mathrm{Off}}$ & 12.1 & 3.4 & 5.1 & 18.2 & \\
\hline & & \% Yield & 33.1 & 6.1 & 22.0 & 44.8 & 22 \\
\hline \multirow[t]{3}{*}{1} & short & OD $\mathrm{On}$ & 38.9 & 7.2 & 28.3 & 55.0 & \\
\hline & & $O D_{\text {Off }}$ & 20.4 & 4.5 & 12.1 & 33.9 & \\
\hline & & $\%$ Yield & 52.3 & 9.3 & 35.2 & 73.0 & 34 \\
\hline \multirow[t]{3}{*}{1} & medium & $\mathrm{OD}_{\mathrm{On}}$ & 44.1 & 4.5 & 37.4 & 51.5 & \\
\hline & & OD ${ }_{\text {Off }}$ & 20.9 & 3.6 & 9.9 & 25.0 & \\
\hline & & $\%$ Yield & 47.9 & 10.6 & 21.2 & 58.9 & 13 \\
\hline \multicolumn{8}{|c|}{$\begin{array}{l}\text { Abbreviations: standard deviation (SD); minimum (Min); maximum (Max); } \\
\text { number of samples }(\mathrm{N}) \text {. }\end{array}$} \\
\hline \multicolumn{8}{|c|}{ Data omitted for samples loaded at >55 OD. } \\
\hline
\end{tabular}

(typically up to $50 \mathrm{OD}$ ) in aqueous ammonium hydroxide (30\% ammonia), were placed into rack 2 . An equivalent number of the following were placed into the racks indicated: $(i)$ packed columns into the sliding assembly above rack 4; (ii) culture tubes $(13 \times$ $100 \mathrm{~mm}$ ) into rack 3; and (iii) round bottom glass vials $(12 \times 55 \mathrm{~mm})$ (Part No. 2954667K; Gilson or Part No. 73500S237; Kimble/Kontes Glass, Vineland, NJ, USA) into rack 4 (for elution) and rack 5 (for quantification). Tables 2 and 3 detail the automated protocols for oligonucleotide purification and quantification.

For the quantification of unpurified oligonucleotides, the protocol is exactly as described in Table 3, except that dried oligonucleotides are placed in rack 2 and redissolved in $2.14 \mathrm{~mL} \mathrm{H}_{2} \mathrm{O}$ air (aspirate at $24 \mathrm{~mL} / \mathrm{min}$ and dispense at $96 \mathrm{~mL} / \mathrm{min}$ ) by bubbling $5 \mathrm{~mL}$ air. Steps 2-6 are identical to the protocol in Table 3, except that in step 3, $2.9 \mathrm{~mL}$ $\mathrm{H}_{2} \mathrm{O}$ are added. Both of the protocols for quantification of oligonucleotides are fully automated.

Solvent reservoirs are as follows: position 0, 0.1 M TEAA, $\mathrm{pH} 7.0$; position $1, \mathrm{ACN}$; position $2,6.5 \%(\mathrm{vol} / \mathrm{vol})$ $\mathrm{NH}_{4} \mathrm{OH}$; position 3, $2 \%$ TFA (vol/vol); position $4,20 \% \mathrm{ACN}$ (vol/vol) and position 5, deionized, distilled $\mathrm{H}_{2} \mathrm{O}$.

For multiple purifications of aliquots of the same oligonucleotide, the appropriate column was reutilized directly, without intervening wash proce- 
dures. Before first use and between purifications of different oligonucleotides, each packed column was washed on the ASPEC robot with $5 \mathrm{~mL}$ of each of the following in sequence (at a flow rate of $3 \mathrm{~mL} / \mathrm{min}$ ): ACN, $0.1 \mathrm{M}$ TEAA, $\mathrm{pH}$ $7.0,20 \% \mathrm{ACN}$ (vol/vol) and ACN.

Analytical HPLC was performed by ion-exchange chromatography on a Mono Q ${ }^{\circledR}$ HR 5/5 column (Amersham Pharmacia Biotech, Piscataway, NJ, USA) in $20 \mathrm{mM} \mathrm{NaOH}$, pH 12.0, using a $0.0-1.5 \mathrm{M} \mathrm{NaCl}$ gradient, on a Dynamax ${ }^{\circledR}$ HPLC System (Varian Instruments) with a Model 234 Autoinjector (Gilson). Buffer A is $20 \mathrm{mM} \mathrm{NaOH}$ in $\mathrm{H}_{2} \mathrm{O}$, and Buffer $\mathrm{B}$ is $20 \mathrm{mM} \mathrm{NaOH}-2$ $\mathrm{M} \mathrm{NaCl}$ in $\mathrm{H}_{2} \mathrm{O}$. The gradient following sample injection is $0 \%-20 \% \mathrm{~B}$ in 4 min, 20\%-60\% B in 20 min, 60\%-75\% $\mathrm{B}$ in $1 \mathrm{~min}$ and $4 \mathrm{~min}$ at $75 \% \mathrm{~B}$. Before the next run, the gradient returns to $0 \%$ $\mathrm{B}$ in $1 \mathrm{~min}$ and equilibrates at $0 \% \mathrm{~B}$ for $4 \mathrm{~min}$. The flow rate is $1 \mathrm{~mL} / \mathrm{min}$.

Statistical analysis was by the Stu-

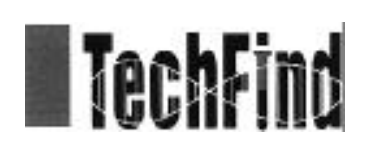

Director of Medicinal Chemistry:

Start-up Biotech Company interested in CNS research needs a Ph.D. with a minimum 5-8 years exp. with a minimum of 2 years solid management experience. Must have proven track record in medicinal chemistry.

\section{Sr. Scientist Synthetic Organic Chemistry:}

Start-up biotech company interested in small molecule drug discovery using novel peptide chemistries needs Ph.D. with a minimum of 2 years industry experience in asymmetric synthesis, chiral chemistry and peptide chemistry.

Resumes to: TECHFIND, Inc. PO Box 626 Natick, MA 01760

Email: resume@techfind.com

Fax: (508) 647-0110

Phone: (508) 647-0111

Table 2. Automated Protocol for Oligonucleotide Purification

1. Pipet $0.9 \mathrm{~mL}$ oligonucleotide, $1.8 \mathrm{~mL} \mathrm{H}_{2} \mathrm{O}$ and $1.5 \mathrm{~mL} 0.1 \mathrm{M}$ triethlylammonium acetate (TEAA), $\mathrm{pH} 7.0$, into a $13 \times 100-\mathrm{mm}$ culture tube (rack 3 ) and mix by bubbling air through the solution.

2. Condition column with $2 \mathrm{~mL}$ acetonitrile (ACN).

3. Wash column with $4 \mathrm{~mL} 0.1 \mathrm{M}$ TEAA, $\mathrm{pH}$ 7.0.

4. Load $4 \mathrm{~mL}$ diluted oligonucleotide solution in rack 3 onto column.

5. Wash column with $19 \mathrm{~mL} 6.5 \% \mathrm{NH}_{4} \mathrm{OH}$ (to remove failure sequences).

6. Wash column with $2.5 \mathrm{~mL} 0.1 \mathrm{M}$ TEAA, $\mathrm{pH} 7.0$.

7. Wash column with $4 \mathrm{~mL} 2 \%$ trifluoroacetic acid (TFA) (to detritylate oligonucleotide).

8. Wash column with $2.5 \mathrm{~mL}$ 0.1 M TEAA, $\mathrm{pH} 7.0$.

9. Wash column with $2.5 \mathrm{~mL} \mathrm{H}_{2} \mathrm{O}$.

10. Wash column with $0.5 \mathrm{~mL} 20 \% \mathrm{ACN}$.

11. Elute oligonucleotide with $1.2 \mathrm{~mL} 20 \%$ ACN into a glass collection tube $(12 \times$ $55 \mathrm{~mm}$ ) (rack 4).

12. Blow out the remaining oligonucleotide solution into the collection vial with $1.5 \mathrm{~mL}$ air.

For oligonucleotide purification, step 1 (oligonucleotide pipetting and mixing) and all aspirations are at a flow rate of $24 \mathrm{~mL} / \mathrm{min}$. Steps 2 and 3 (column conditioning/equilibration) and steps 5-10 (column washing) are at a flow rate of $3 \mathrm{~mL} / \mathrm{min}$. Step 4 (oligonucleotide loading to column) is at a flow rate of $1.5 \mathrm{~mL} / \mathrm{min}$. Step 11 (oligonucleotide elution) is at a flow rate of $3 \mathrm{~mL} / \mathrm{min}$ and bubbling with air is at a flow rate of $6 \mathrm{~mL} / \mathrm{min}$. For the oligonucleotide purification protocol, the robot performs all steps without user intervention.

Table 3. Automated Protocol for Quantification of Purified Oligonucleotides

1. Mix the eluted oligonucleotide by bubbling $3 \mathrm{~mL}$ air.

2. Transfer $100 \mu \mathrm{L}$ eluted oligonucleotide from rack 4 to a glass collection tube $(12 \times 55 \mathrm{~mm})($ rack 5$)$.

3. Add $900 \mu \mathrm{L} \mathrm{H}_{2} \mathrm{O}$. Mix by bubbling $3 \mathrm{~mL}$ air through the solution.

4. Inject $650 \mu \mathrm{L}$ diluted oligonucleotide to the UV spectrophotometer, and automatically read the OD at $260 \mathrm{~nm}$.

5. ODs can be recorded off the spectrophotometer screen or printed to an ink-jet printer (Cannon Instruments, State College, PA, USA).

For oligonucleotide quantification, aspiration rates are at $24 \mathrm{~mL} / \mathrm{min}$ for all steps except step 4, which aspirates at a rate of $6 \mathrm{~mL} / \mathrm{min}$. Dispense rates are as follows: step 2, $24 \mathrm{~mL} / \mathrm{min}$; step 3, $48 \mathrm{~mL} / \mathrm{min}$ and step 4, $12 \mathrm{~mL} / \mathrm{min}$.

dent's $t$ test, with $p<0.01$ taken as statistically significant difference between means. Any unreported methods were as reported in Ivanetich et al. (4).

\section{RESULTS AND DISCUSSION}

The protocol reported here has been performed on several hundred synthetic oligonucleotides. Data was collected for approximately 330 oligonucleotides for percentage yield, OD loaded onto the cartridge $\left(\mathrm{OD}_{\mathrm{on}}\right)$ and $\mathrm{OD}$ of the product eluted from the cartridge $\left(\mathrm{OD}_{\text {off }}\right)$. The data were correlated with synthesis scale, oligonucleotide length and the number of times that the purification column was used. Reported data include the mean, standard deviation, minimum, maximum and $n$ values.

New columns, and columns previ- 
ously used once or twice, provided identical $\mathrm{OD}_{\text {off }}$ and percentage yields for short (15-25-mers) or medium-length (26-40-mers) oligonucleotides synthesized at the 40-nmol scale. Equivalent results were obtained for the 0.2 - and 1$\mu$ mol scales (data not shown). Because there were no significant differences for new and used columns for equivalent oligonucleotide loads, the data for new and used columns were combined for each synthesis scale. Table 1 presents a summary of the data.

Oligonucleotide lengths were between 15- and 69-mers, with most data collected for the purification of 15-40mers. For short oligonucleotides (1525-mers), $\mathrm{OD}_{\text {on }}$ was approximately 10 ODs for oligonucleotides synthesized at the $40 \mathrm{nmol}$ scale of synthesis. The corresponding value for oligonucleo- tides synthesized at the 0.2 and $1 \mu \mathrm{mol}$ scales were 27 and 39 ODs, respectively. For short oligonucleotides, the $\mathrm{OD}_{\text {off }}$ for the $40 \mathrm{nmol}$ scale was approximately 2.7 ODs vs. OD off $_{\text {of ap- }}$ proximately 11 and 20 ODs for the 0.2 and $1 \mu \mathrm{mol}$ scales of synthesis. The percentage yield was $28 \%$ for the 40 nmol synthesis scale and $43 \%$ and $53 \%$ for the 0.2 and $1 \mu \mathrm{mol}$ synthesis scales.

For medium oligonucleotides (2640-mers), $\mathrm{OD}_{\text {on }}$ was approximately 13 ODs for oligonucleotides synthesized at the $40 \mathrm{nmol}$ scale of synthesis. The corresponding value for oligonucleotides synthesized at the 0.2 and $1 \mu \mathrm{mol}$ scales were 36 and 44 ODs. For medium-length oligonucleotides, the $\mathrm{OD}_{\text {off }}$ for the $40 \mathrm{nmol}$ scale was approximately 3.3 ODs vs. OD off $_{\text {of approximately }}$ 12 and 21 ODs for the 0.2 and $1 \mu \mathrm{mol}$

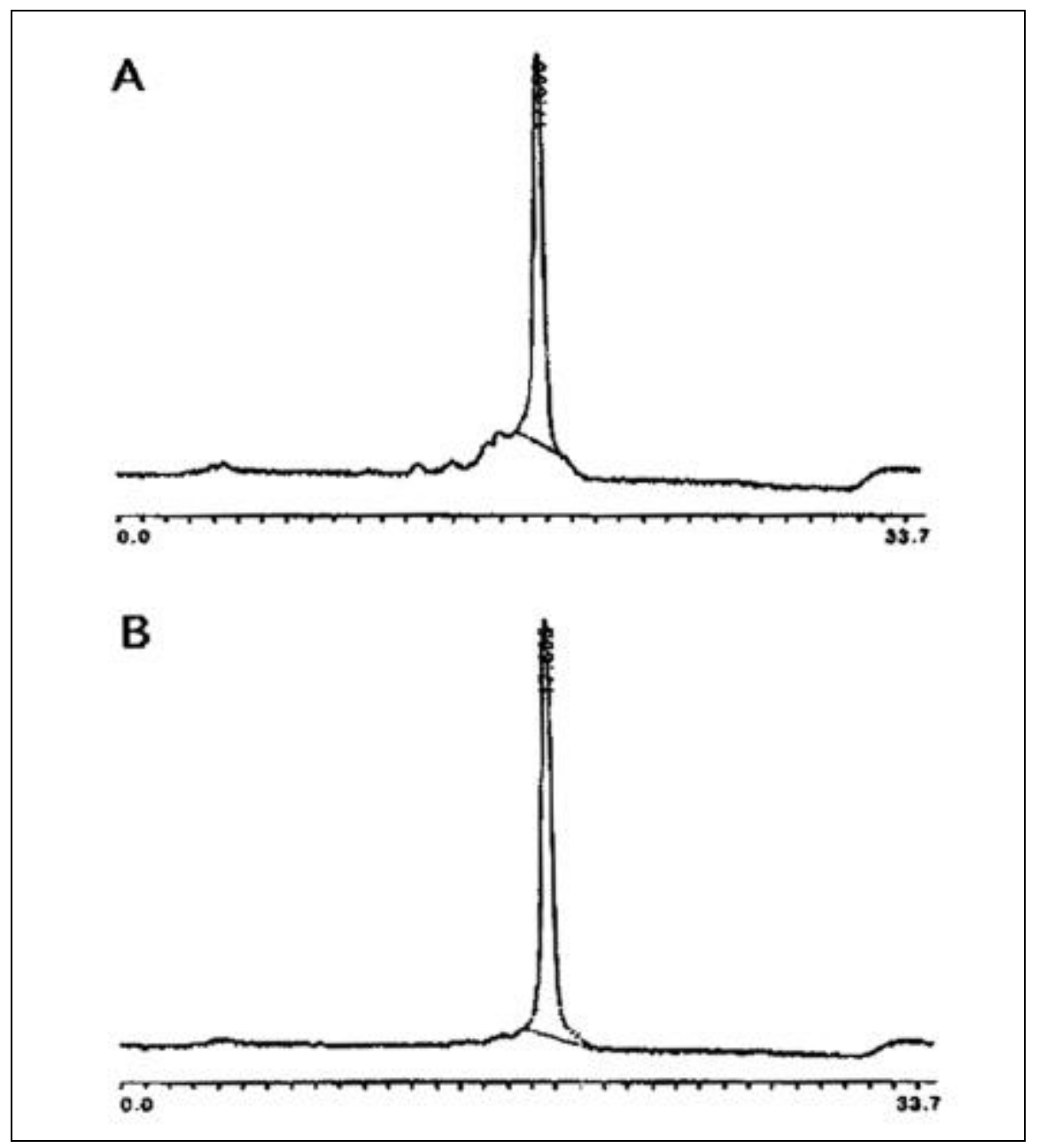

Figure 1. HPLC Chromatograms of a 30-mer oligonucleotide. (A) Crude detritylated oligonucleotide. (B) Oligonucleotide purified by Amberchrom method. Chromatography was performed on a Mono Q ion-exchange column. See Materials and Methods for experimental details. 
scales of synthesis, respectively. The percentage yield was approximately $26 \%$ for the $40 \mathrm{nmol}$ synthesis scale vs. $33 \%$ and $48 \%$ for the 0.2 and $1 \mu \mathrm{mol}$ synthesis scales. For short or mediumlength oligonucleotides, the $\mathrm{OD}_{\mathrm{on}}$, $\mathrm{OD}_{\text {off }}$ and percentage yield were 1.3to 7.5 -fold greater $(p<0.001)$ for oligonucleotides synthesized at the 0.2 or $1 \mu \mathrm{mol}$ scales compared with oligonucleotides synthesized at the $40 \mathrm{nmol}$ scale. For all three parameters, the data for the $1 \mu \mathrm{mol}$ scale was significantly greater than that for the $0.2 \mu \mathrm{mol}$ scale $(p<0.01)$.

The maximum capacity of the column was estimated to be approximately $45-50$ ODs (up to ca. $200 \mathrm{nmol}$ ). This was concluded from an examination of $\mathrm{OD}_{\text {on }}$ vs. percentage yield, in which oligonucleotides loaded with $>50$ ODs typically exhibited lower than average percentage yields (data not shown). This is not an exact capacity because ODs provide only an approximate measurement of oligonucleotide concentration, and there is some variation (ca. 5\%) in the amount of resin packed into each column.

A 30-mer oligonucleotide was synthesized trityl-on. A portion was detritylated, and a second portion was subjected to purification by the reported method. Figure 1 provides analytical HPLC profiles of the same detritylated crude and purified 30-mer oligonucleotide. For oligonucleotides under 40-50-mers, the quality of the purified oligonucleotides typically equates to other cartridge methods or HPLC methods. For longer oligonucleotides, we recommend a second purification by a method such as HPLC to remove side products arising from heterogeneity of the trityl-on species and/or non-tritylated failure sequences not fully removed by the cartridge purification method. For oligonucleotides purified on previously used columns, there was no evidence of cross contamination by the previously purified oligonucleotide (Figure 2). There was no evidence of experimental artifacts from oligonucleotides purified on previously used columns for over 920 oligonucleotides purified by this method and used for polymerase chain reaction (PCR), automated DNA-sequencing primers and other types of experiments. Typically, columns were used for a total of three purifications before being discarded. As mentioned above, the percentage yield was not affected by reuse of the column.

We performed a series of control experiments, mixing trityl-on and trityloff oligonucleotides in various ratios to assess the ability of the protocol to separate trityl-off failure products from the full-length, trityl-on oligonucleotide.

One experiment was performed with a pair of short oligonucleotides (20mers); the experiment was repeated with a pair of medium-length oligonucleotides (44-mers). Within each pair, the oligonucleotides were chosen so that the trityl-off oligonucleotides had significant differences in retention time (ca. 1.5-5 $\mathrm{min}$ ) on Mono Q ion-ex-

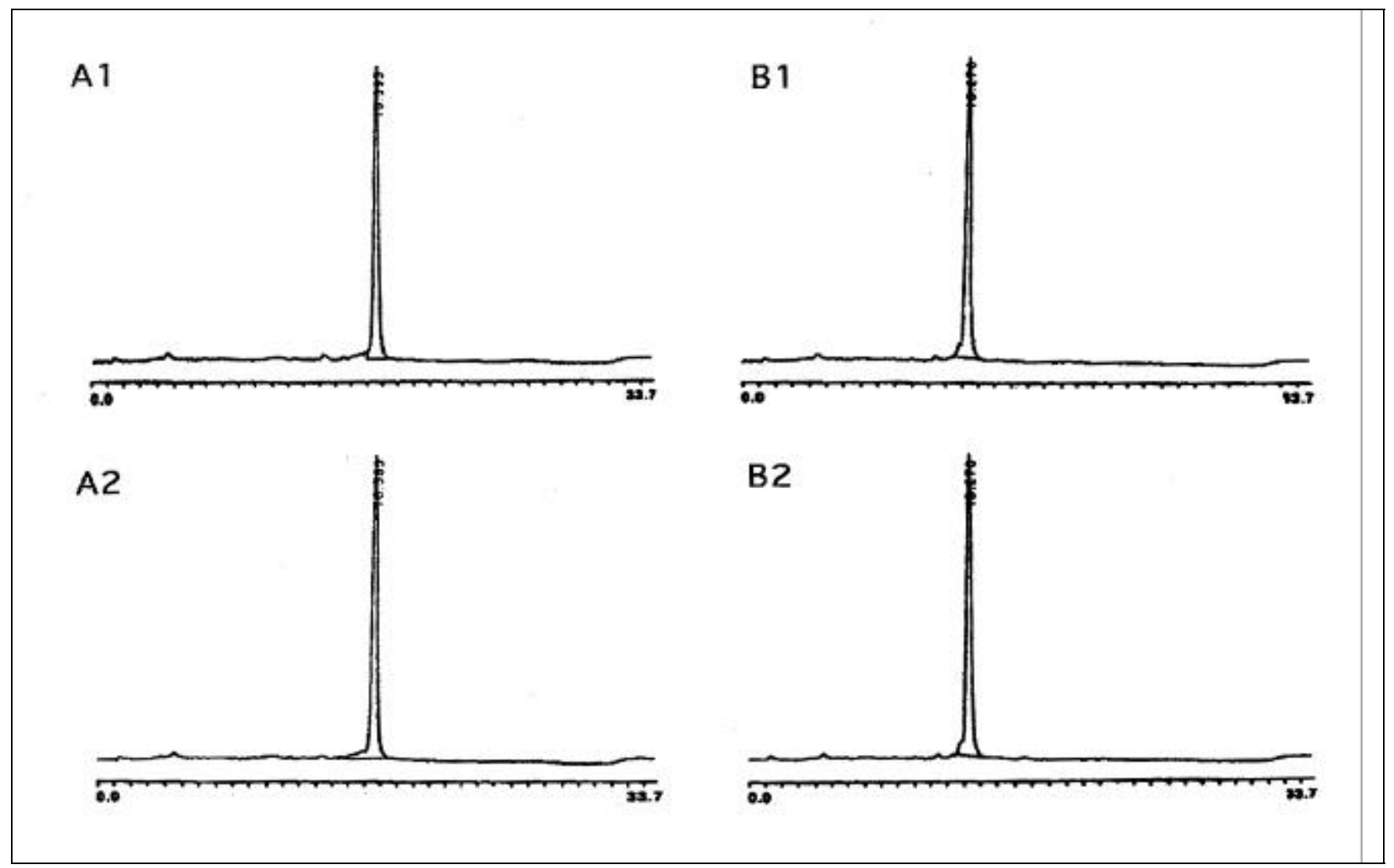

Figure 2. Comparison of oligonucleotides purified on new vs. used columns. (A1) 15-mer (retention time 16.4 min) purified on new column. (A2) The same 15 -mer as in A1 purified on column previously used to purify a 20-mer (retention time $13.3 \mathrm{~min}$ ). (B1) A 20-mer (retention time 13.3 min) purified on new column. (B2) The same 20-mer as in B1 purified on column previously used to purify a 15-mer (as in A1). 
change HPLC (see Materials and Methods). Oligonucleotide pairs were mixed at the ratios of 90:10 or 80:20 of tritylon:trityl-off oligonucleotide. ODs were used as a measure of concentration. For the 20-mers, no significant contamination by the trityl-off species was noted in the final product at initial ratios of $80: 20$ or 90:10 trityl-on:trityl-off oligonucleotide (data not shown).

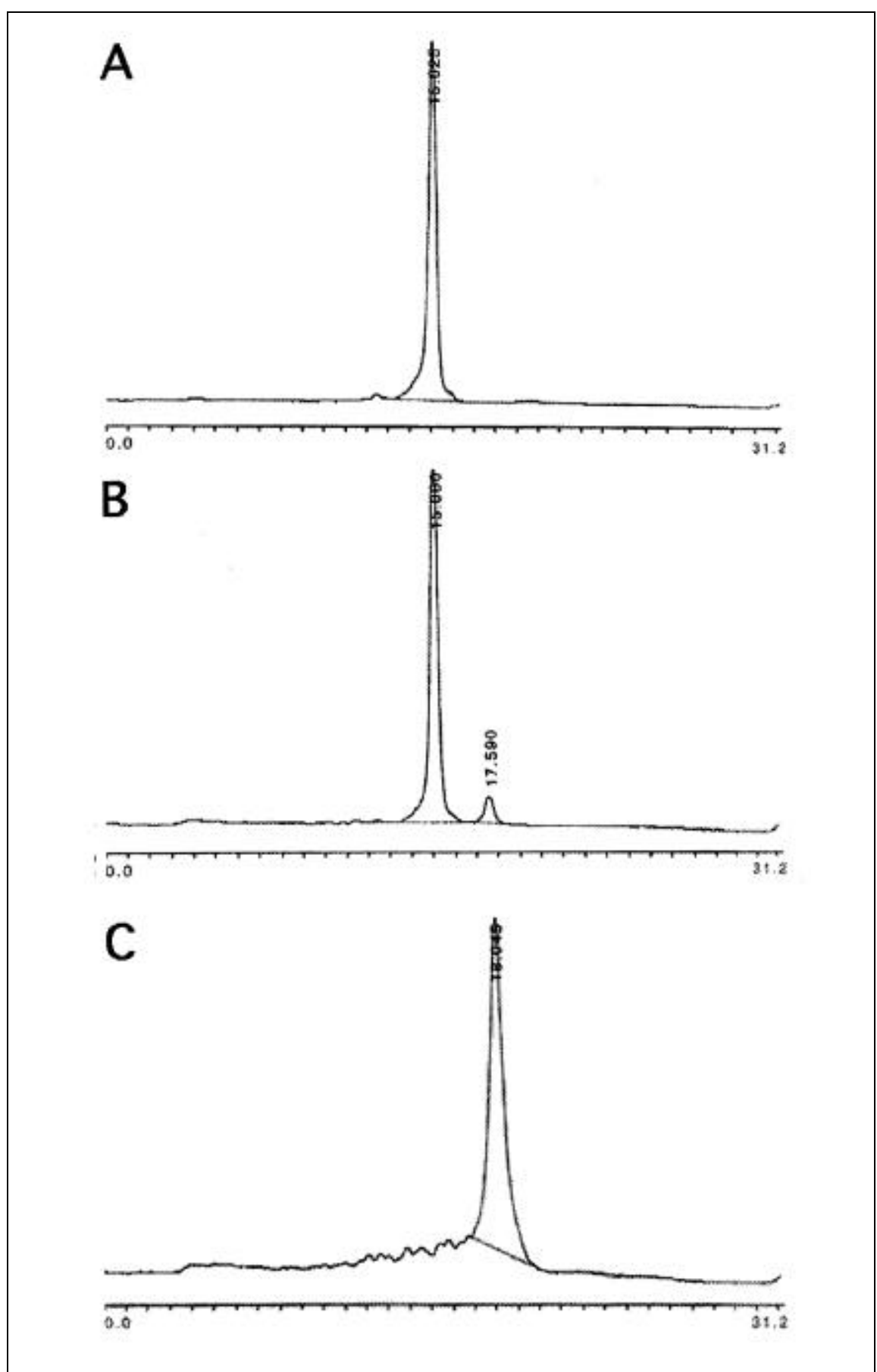

Figure 3. HPLC Chromatograms of a pair of trityl-off and trityl-on, medium-length oligonucleotides (44-mers). (A) Product from the purification of a 90:10 ratio of trityl-on oligonucleotide:trityloff oligonucleotide. (B) Product from the purification of an 80:20 ratio of trityl-on oligonucleotide:trityloff oligonucleotide. (C) Unpurified trityl-off oligonucleotide. Trityl-on oligonucleotide: 44-mer with base composition: A7, C5, G18, T14, $1.96 \mathrm{nmol} / \mathrm{OD}_{260}$. Trityl-off oligonucleotide: 44 -mer with base composition: A10, C14, G8, T12, $2.09 \mathrm{nmol} / \mathrm{OD}_{260}$. HPLC was as in Figure 1. 
Because the overall yield of the tritylon oligonucleotide ranged from $32 \%-$ $36 \%$, it appeared that the trityl-off species did not interfere with the purification. For the 44-mers, the purified product from the 90:10 ratio mixture also provided no evidence of contamination of the final product (retention time $15 \mathrm{~min}$ ) with the trityl-off species (retention time $17.8 \mathrm{~min}$ ). However, at an initial ratio of trityl-on:trityl-off for 44-mer oligonucleotides of 80:20, the final product was contaminated by the trityl-off species to the extent of approximately $7 \%$ (Figure 3 ).

To assess the quality of the purification method (i.e., how well the method removed non-tritylated failure sequences), two trityl-off oligonucleotides, a 20-mer and a 39-mer, were purified by the standard Amberchrom protocol. For each oligonucleotide, 11.5 OD were loaded onto the cartridge. The yield of the 20 -mer was $0.6 \mathrm{OD}$ or $5.2 \%$ and the yield of the 39-mer was 1.0 OD or 8.6\%. Analytical HPLC confirmed that the apparent product exhibited a HPLC profile typical of an oligonucleotide, indicating that the apparent product was not a nonoligonucleotide artifact. The longer detritylated oligonucleotide appeared to bind more strongly to the Amberchrom resin, because a higher percentage was eluted in the fraction that would contain the final product. A similar experiment was performed with Oligopure ${ }^{\mathrm{TM}}$ Cartridges (Hamilton, Reno, NV, USA) using the manufacturer's protocol. Twenty ODs of a non-tritylated 31-mer oligonucleotide were loaded, and a yield of 3.5 OD or $18 \%$ was achieved, suggesting that higher contamination of purified species with non-tritylated oligonucleotides-presumably equivalent to failure sequences-would be observed with Oligopure cartridges than with the Amberchrom method.

We compared the ODs for oligonucleotides diluted and measured automatically on the ASPEC with the same oligonucleotide diluted manually and the ODs measured manually on a Model 8451A Diode Array Spectrophotometer (Hewlett-Packard, Wilmington, DE, USA). There was no significant difference between the data sets (data not shown).

We assessed the reproducibility of OD measurements on the UV spectrophotometer for undiluted oligonucleotide samples and oligonucleotide samples diluted by the appropriate program on the ASPEC. The reproducibility of

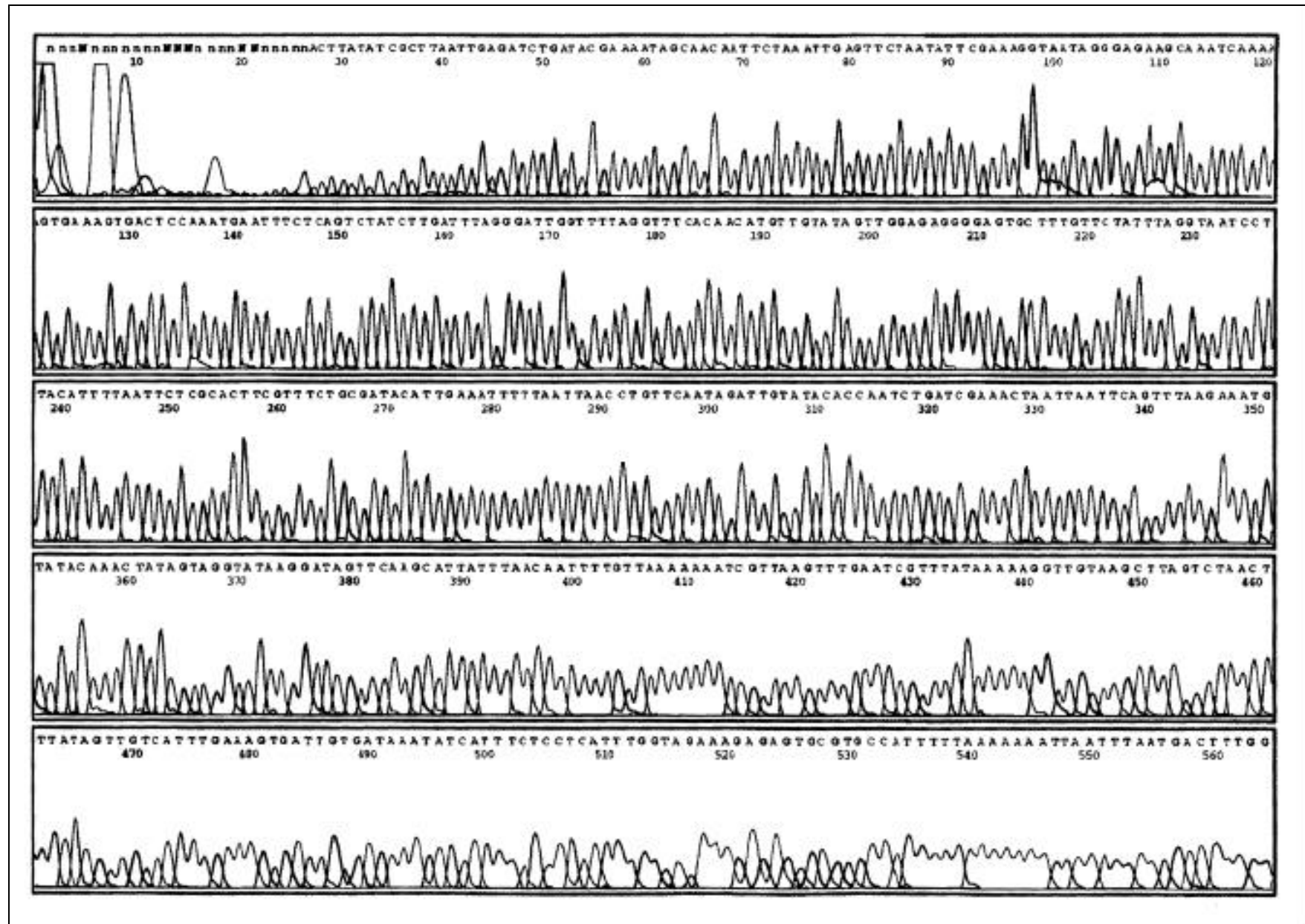

Figure 4. A typical successful DNA sequencing run on a custom template with a primer purified by the reported method. The sequencing reaction was performed using the ABI Big Dye terminator chemistry, according to the manufacturers specifications, and the reacted sample was run on a Model 377 DNA sequencer. The primer was a 17-mer, with base composition: A6, G4, C4, T3. 
OD measurements (ODs of 0.1 or 0.2 ) on the UV spectrophotometer for undiluted samples following direct injection into the spectrophotometer by the ASPEC was found to be high (coefficient of variance $[\mathrm{cv}]=0.007-0.009$, two data sets, $n=3$ each).

The reproducibility and accuracy of the dilutions on the Gilson ASPEC were determined using the automated protocols for quantification of purified and crude oligonucleotides (see Materials and Methods) on solutions of a synthetic oligonucleotide standard 20-mer primer in $\mathrm{H}_{2} \mathrm{O}$ (initial concentrations ranged from $1-6 \mathrm{OD} / \mathrm{mL}$; final concentrations ranged from $0.1-0.2 \mathrm{OD} / \mathrm{mL}$ ). The protocols for the quantification of purified and crude oligonucleotides utilize $1: 10$ and $1: 30$ dilutions of $100 \mu \mathrm{L}$ oligonucleotide solution. Both methods provided acceptable reproducibility; the $\mathrm{cv}$ of the measurements varied 0.0010.009. Comparable cv on the diode array spectrophotometer using manual dilution ranged from 0.003-0.030.

Accuracy was maintained by periodic determination of a standard curve, with incorporation of resulting correction factors into the UV spectrophotometer readings.

The reported purification method has several advantages over the previously reported automated method based on Nensorb resin (4). The Amberchrom CG-50 resin has higher capacity than the Nensorb resin, and thus requires less resin per column (i.e., 0.3 g Amberchrom CG-50 vs. 2 g Nensorb resin). The cost of the Amberchrom resin is $\$ 0.77$ per column vs. $\$ 3.10$ per column for Nensorb resin. Thus, the Amberchrom resin provides a cost savings of $\$ 2.33$ per column. The reported protocol also requires less buffer per run than the Nensorb protocol. Solvent consumption (excluding water) for column conditioning and oligonucleotide purification was $22 \mathrm{~mL}$ for the Amberchrom method, compared to $38 \mathrm{~mL}$ for the Nensorb method. Correspondingly, the wash protocol for the Amberchrom resin uses $12 \mathrm{~mL}$ of solvent, whereas $20 \mathrm{~mL}$ is needed for the Nensorb protocol. In addition, it was observed that columns packed with Amberchrom CG-50 could be used for three purification runs with the same packing, without loss of yield or measurable cross contamination of the oligonucleotides. For the Amberchrom method, the total cost per run including reagents and consumables is $\$ 2.30$ for a single use of the packed column. If the column is used for three oligonucleotide purifications, the cost is $\$ 1.09$ per run, for all new reagents, tubes and collection vials per run, but with reuse of the column, resin, frits and sealing inserts.

Furthermore, the new Amberchrom method requires less personnel time than the Nensorb method. Personnel time is decreased because the dilution and mixing of the oligonucleotide and the quantification have been automated. In addition, the final speed-vac, drydown step for reduction of the volume of oligonucleotide eluate in the Nensorb protocol is not needed in the Amberchrom method. Because the final elution solvent was changed from aqueous methanol for the Nensorb method to aqueous ACN for the Amberchrom protocol, for most purposes, oligonucleotides can now be delivered to customers directly without removal of the organic solvent. For the Amberchrom method, oligonucleotides are delivered in $20 \% \mathrm{ACN}$, as suggested in PE Biosystems user bulletins $(9,10)$. Our laboratory has not experienced any problems from purified DNA-sequencing primers delivered in $20 \% \mathrm{ACN}$ for over 200 oligonucleotides.

We compared the purification of oligonucleotides by the Nensorb and Amberchrom methods for the $0.2 \mu \mathrm{mol}$ scale of oligonucleotide synthesis. The data for short and medium-length oligonucleotides synthesized at the 0.2 $\mu$ mol scale of synthesis were combined for comparison to the Nensorb data. The Amberchrom method $(n=47)$ gave a lower percentage yield per run (37\%) than Nensorb $(71 \% \pm 22 \%)$. However, the Amberchrom columns exhibited approximately $300 \%$ higher capacity (45-50 ODs) than Nensorb (capacity 15 ODs), although the former contained only $15 \%$ the weight of resin of the latter (4). Overall, the Amberchrom and Nensorb methods gave approximately equivalent total yields for one purification run (i.e., ca. 12 ODs for Amberchrom vs. 10 ODs for Nensorb).

To determine the $\mathrm{cv}$ for the Amberchrom purification method, eight oligonucleotides (20-27-mers) were 

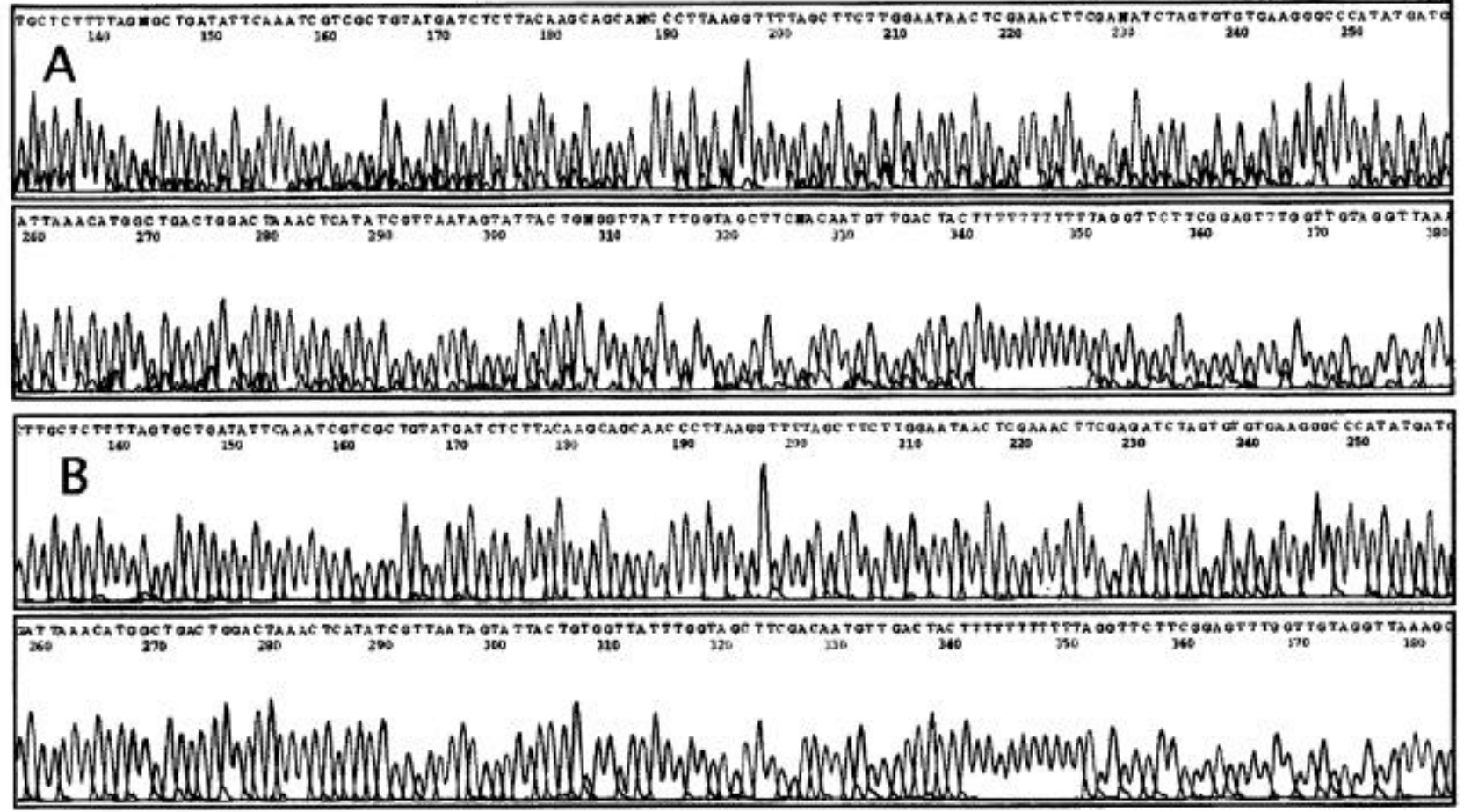

Figure 5. DNA sequencing results on a single nonstandard template with Big Dye chemistry run on a Model 377 DNA sequencer using the same purified primer. Primer was a 20-mer with base composition: A5, G9, C3, T3. (A) Primer was synthesized and purified by a commercial vendor. (B) Primer was synthesized by this laboratory and purified by the reported method.

synthesized at the $1 \mu \mathrm{mol}$ scale and aliquoted into 4 or 5 portions. The aliquots were purified on new columns, columns used once or columns used twice. The overall yields for the new columns ranged from $37 \%-56 \%$. For new columns, the cv ranged from 0.04-0.12 and averaged 0.09. This was lower than the cv for the Nensorb robotic method, which was 0.13 (4).

Over 200 primers purified by the Amberchrom method have been used for automated DNA sequencing on Models 373S, 377 and 377-96 DNA sequencers, using Rhodamine, dichlororhodamine and Big Dye ${ }^{\mathrm{TM}}$ terminator chemistries (all from PE Biosystems). Figure 4 shows a typical successful run with a custom plasmid template and custom primer purified by this method; the sequence was run on a Model 377 DNA sequencer using the Big Dye terminator chemistry. For this run, the first ambiguous base was at position 672 . The total number of uneditable/ambiguous bases was 1/700.

Figure 5 shows a comparison of the sequencing data obtained from one custom plasmid template sequenced using the Big Dye chemistry with a primer synthesized and purified by a cartridge method by a commercial vendor (Figure 5A) or a primer of the identical sequence synthesized inhouse and purified by the reported method (Figure 5B). For these sequencing runs, the first uneditable miscall (this excludes the first 40 bases of the sequence, which can be obscured by unincorporated dye blobs and other problems unrelated to primer quality) or $n$ was at base 62 for Figure $5 \mathrm{~A}$ and base 580 for Figure 5B. The number of uneditable $n$ in 500 bases was 14 for Figure 5A and zero for Figure 5B. These criteria confirm how well the reported method works for automated DNA-sequencing compared to the identical primer synthesized and purified by an alternative cartridge method. Analogous differences in sequence quality for custom templates were seen for rhodamine-termination chemistry for primers from our laboratory vs. those from a commercial vendor (data not shown).

In our experience, the need for $\mathrm{pu}$ rification of oligonucleotide primers for automated DNA sequencing is a function of the quality of the synthesis, the difficulty of the template and the DNAsequencing chemistry. We have observed that when oligonucleotide quality is poor, automated DNA sequencing benefits from purification of the oligonucleotide primer. This is more striking for difficult templates. Because it can be difficult to troubleshoot DNA-sequencing problems in a biotechnology core facility, in which users submit a wide variety of primers and templates of variable quantity and quality, our core laboratory requests that users submit purified primers so that we can eliminate one cause of potential sequencing problems. With purified primers, plus inhouse quantification of templates, this laboratory achieves over an $85 \%$ success rate for DNA sequencing.

Protocols are available in hard copy, by e-mail or on disk. 


\section{ACKNOWLEDGMENTS}

We wish to thank Hugh Mackie and Robert Somers of Glen Research for assistance during the development of the protocol.

\section{REFERENCES}

1.Ausserer, W.A. and M.L. Biros. 1995. Highresolution analysis and purification of synthetic oligonucleotides with strong anion-exchange HPLC. BioTechniques 19:136-139.

2.Becker, C., J.W. Efcavitch, C.R. Heiner and N.F. Kaiser. 1985. Use of a C4 column for reversed-phase high performance liquid chromatographic purification of synthetic oligonucleotides. J. Chromatogr. 326:293-299.

3.Germann, M.W., R.T. Pon and J.H. van de Sande. 1987. A general method for the purification of synthetic oligodeoxyribonucleotides containing strong secondary structure by reversed-phase high-performance liquid chromatography on PRP-1 resin. Anal. Biochem. 165:399-405.

4.Ivanetich, K.M., J. Akiyama, D.V. Santi and M. Reschenberg. 1991. Automated purification of synthetic oligonucleotides. BioTechniques 10:704-707.

5.Johnson, B.A., S.G. McClain, E.R. Doran, G. Tice and M.A. Kirsch. 1990. Rapid purification of synthetic oligonucleotides: a convenient alternative to high-performance liquid chromatography and polyacrylamide gel electrophoresis. BioTechniques 8:424-429.

6.Lo, K.-M., S.S. Jones, N.R. Hackett and H.G. Khorana. 1984. Specific amino acid substitutions in bacterioopson: replacement of a restriction fragment in the structural gene by synthetic DNA fragments containing altered codons. Proc. Natl. Acad. Sci. USA 81:22852289.

7.Maniatis, T., A. Jeffrey and $H$. van deSande. 1975. Chain length determination of small double- and single-stranded DNA molecules by polyacrylamide gel electrophoresis. Biochemistry 14:3787-3794.

8.McBride, L.J., S. McCollum, S. Davidson, J.W. Efcavitch, A. Andrus and S.J. Lombardi. 1988. A new, reliable cartridge for the rapid purification of synthetic DNA. BioTechniques 6:362-367.

9.PE Biosystems Application Note. 1996. Dye terminator cycle sequencing with purified primers from the ABI 3948 show beneficial results. PE Biosystems, Foster City, CA.

10.PE Biosystems 3948 Quick Reference Guide. 1996. A guide to using primers made on the ABI 3948 synthesis and purification system. PE Biosystems, Foster City, CA.

11.Scanlon, D., J. Haralambidis, C. Southwell, J. Turton and G. Tregear. 1984. Purification of synthetic oligodeoxyribonucleotides by ion-exchange high-performance liquid chromatography. J. Chromatogr. 336:189-198.

12.Zieske, L.R. 1988. Novel approaches in synthetic polynucleotide purification. BioChromatography 3:112-117.
13.Zon, G. and J.A. Thompson. 1986. A review of high-performance liquid chromatography in nucleic acids research. II. Isolation, purification, and analysis of oligodeoxyribonucleotides. BioChromatography 1:22-31.

Received 22 March 1999; accepted 21 June 1999.

\section{Address correspondence to:}

Kathryn M. Ivanetich

Biomolecular Resource Center

Box 0541

University of California, San Francisco

San Francisco, CA 94143, USA

Internet: kathyi@cgl.ucsf.edu 